\title{
Chapter 19 \\ Lessons and Future Perspectives \\ of Earth Observation and GIT \\ in the HKH
}

\author{
Mir A. Matin, Birendra Bajracharya, and Rajesh Bahadur Thapa
}

\subsection{Introduction}

During the last decade, SERVIR has been striving for realizing its vision of "Space to Village" by implementing services that provide innovative solutions to improve livelihoods and foster self-reliance with the help of EO and geospatial technologies (Chap. 1). Over these years, there has been significant development in the field of EO and geospatial technology. However, the capacity of the key agencies to utilize these advancements to produce, disseminate, and use information has not been able to catch up with these developments. As cited in the previous chapters, SERVIR-HKH has been working with various partners and stakeholders in co-developing and implementing applied, user-driven EO and geospatial information services in the HKH region. SERVIR-HKH recognizes that the sustainability of information products and applications and their use requires an understanding of users and their needs. Understanding the user's needs and organizational context is the key to delivering effective services. As illustrated in Chaps. 2 and 3, the needs assessment study revealed that the use of geospatial data in the region started in the early 1990s, but there are still gaps in the institutionalization and sharing of information. Often, individual agencies produce geospatial information for their own purpose and do not share it due to lack of policies. Besides, in most cases, the information would have been generated through specific projects funded by external agencies without proper sustainability planning. And as has happened in many cases, those services could not be continued due to lack of resources and capacity.

SERVIR-HKH has gone through a continuous learning process to improve and innovate the service design and delivery approach. From the experiences of the first phase, it was realized that systematic service planning was required to define the roles of the partners at different stages of service development, delivery, and

M. A. Matin $(\bowtie) \cdot$ B. Bajracharya $\cdot$ R. B. Thapa International Centre for Integrated Mountain Development, Kathmandu, Nepal e-mail: mir.matin@icimod.org

(C) The Author(s) 2021

B. Bajracharya et al. (eds.), Earth Observation Science and Applications for Risk Reduction and Enhanced Resilience in Hindu Kush Himalaya Region, https://doi.org/10.1007/978-3-030-73569-2_19 
maturity. The service planning approach (Chap. 2) adopted by SERVIR facilitates a clear understanding of the implementation pathway of service from conceptualization to sustainable adoption by the partners. Well-designed toolkits to provide guidance at each step of service planning, including needs assessment, design, monitoring, and delivery, supported by a strategy for user engagement, communication, gender, capacity development, and $\mathrm{M} \& \mathrm{E}$ have been the fundamental aspects of successful implementation of the service planning approach. The specific focus on gender and social inclusiveness were important to ensure that the services were beneficial for the marginalized populations. The participatory impact pathway and the theory of change, together with the monitoring and evaluation process, have also been helpful in keeping the process on track to achieve the expected outcome.

Institutional arrangements with partners are critical to their engagement in the development and implementation process. In this regard, the systematic capacity building of the partners to use the applications and interpret the information products is necessary. The support from subject matter experts and the Applied Science Team from US universities and institutes also enabled to build the capacity of the regional hub and develop information solutions to cater to the users' needs. Further, the global SERVIR network led by NASA facilitated the incorporation of the latest methods in data analysis and system development; while the integration of NASA's applied science project with the service design process enabled the hub team to use state-of-the art methodology and build the capacity of the team and the partner agencies.

In this chapter, we look at the key lessons that have been learnt and the future directions of the EO applications through initiatives like SERVIR.

\subsection{Lessons from the Thematic Services}

As described in earlier sections, SERVIR-HKH prioritized four broad service areas: agriculture and food security; land use, land cover change, and ecosystems; water and hydro-climatic disasters; and weather and climate. The specific services were developed through recommendations after the needs assessment stage. Although a common approach of service planning was used in service design and development, each thematic area in different countries had their specific needs and challenges. In the following sections, we present our experiences in implementing the service in the different service areas.

\subsubsection{Agriculture and Food Security}

SERVIR-HKH has focused on drought monitoring and crop area mapping as two of its primary services (Chaps. 4 and 5). Early assessment of the cereal crop area and production helps in providing critical information in terms of managing food 
security. In this context, the availability of EO images from the optical and SAR sensor with high spatial and temporal frequency enables the assessment of crop area and assists in monitoring crop health across a large geographic area, thereby supplying critical insights into potential production loss. Also, the availability of free cloud-based access and processing platforms like GEE opens up more opportunities to develop a standard automatic data-processing system that reduces dependence on highly skilled human resources and computing hardware. The work with the Afghanistan Ministry of Agriculture, Irrigation, and Livestock (MAIL) for developing a wheat-area mapping system is an example of establishing one such system. The collaboration with the Bangladesh Agricultural Research Council for mapping winter rice in the country has also delivered promising results.

While the automation of the data-processing system reduces the requirement of highly skilled geospatial professionals in the respective agencies, there is still a need for some field data collection for training and validation of the image classification results. SERVIR-HKH has developed mobile applications to standardize the field data collection process. With a short training, such a system could successfully be used for collecting field data. For example, we have trained the staff of the field agencies of Nepal's Ministry of Agriculture and Livestock Development (MoALD) virtually (due to the COVID-19 pandemic), and they have gone on to conduct such fieldwork. This online arrangement made it possible to carry out the activities effectively and on time, even when the movement was limited due to the pandemic.

Meanwhile, the RS-based crop area mapping has been done with more than $80 \%$ accuracy. This accuracy could be improved through the estimation of statistical uncertainty and bias adjustment using ground samples. The combination of RS-based mapping and bias correction models could provide the most accurate estimate of the crop area. While an RS-based crop area map provides a good indication as to whether there has been any potential deviation from the average yield, there is still a need for a quantitative forecast of the actual yield. The assessment of the crop area does provide a good understanding of crop production, but a more in-depth understanding of the potential yield is important for better management of food security. In this regard, the satellite-based NDVI monitoring of crop growth is a good tool to analyze the deviations in greenness at different growth stages. This information is adding more insights into assessing the condition of crops and gives prior signals about any yield loss.

Traditionally, crop yield is estimated using the crop-cut survey in which systematic samples are collected from the crop fields. Yield estimation could be done either empirically using statistical or machine learning model or using process based models. The crop area from remote sensing together with historical yield data could be used for the development of empirical yield model. The crop area and the input from the drought monitoring model could be integrated to develop process-based yield model. Monitoring drought-related stress on crops at different stages of their growth can also help in managing irrigation and thereby reduce the risk of production loss. 
While a seasonal drought warning system can provide important information that can be used for agriculture planning, the uncertainties involved in such a prediction and the lack of understanding about those uncertainties by the farmers limit the use of such a system. Thus, capacity building of the agriculture extension workers and farmers in the interpretation and effective use of the drought forecasting system is vital for the broader uptake of drought predictions.

\subsubsection{Land Use, Land Cover Change, and Ecosystem}

Land cover mapping is one of the oldest applications of remote sensing. Over the last decades, the HKH countries implemented many land cover mapping projects with funding and technical support from different international agencies. Depending on the funding sources and the available images and expertise, these projects used different data sources and methodologies for land cover mapping. But these land cover maps, due to their differences in resolution, classification schema, and class definition, are not suitable for change analysis which is required for various reporting purposes. The methodology used for land cover map generation was also rather tedious and producing land cover maps for multiple years was time consuming and costly. With the progress in cloud-based access to time series satellite images and processing platforms, land cover maps can be generated relatively easily for all the countries within HKH. For example, the archive of Landsat images with GEE was instrumental in developing the Regional Land Cover Monitoring System (RLCMS) algorithm and tools (Chap. 6) to produce annual land cover maps for the years 2000-2018 for the entire HKH region. Meanwhile, SERVIR-HKH has also helped develop country-level systems for Afghanistan, Bangladesh, Myanmar, and Nepal in partnership with the corresponding national departments.

While RLCMS has provided an approach and process for consistent time series land cover data, there have been some limitations and challenges beyond the control of the system development team. While the land cover maps for earlier years suffers from lack of quality reference data, the maps produced via the new method do not match with the legacy data or the published statistics; so, government agencies are hesitant to adopt the new data. While developing land cover maps, it is important that all the potential users of the data sets understand and agree with the definition of different land cover classes. However, despite all the efforts to develop a classification schema with the unambiguous class definition, there are some classes where the users do not agree on the definition.

The lack of adequate human resources and frequent transfer of officials have been some of the key challenges in the sustainable use of the new mapping method. While the process of co-development of the national land cover maps for Afghanistan and Nepal has built the capacity of the partner agencies, it is important to have regular feedback and the mechanism of backstopping to sustain the production of future land cover maps. Also, the Landsat-based maps are not adequate for more detailed classification of some land covers classes; this can be improved 
by using higher-resolution images like Sentinel II or through the fusion of images from multiple sensors. Besides, the improvements in the machine learning algorithms can be used for enhancing the accuracy of the existing classes or the inclusion of new ones.

While RLCMS provides information on the forest cover extent and the change dynamics that are useful for monitoring deforestation, in the HKH region, forests are also going through degradation, resulting in a thinning of canopy density, loss of biodiversity, and increase in the occurrence of invasive plants (this is particularly critical in the case of Nepal). Degradation happens due to both anthropogenic and natural processes. To address this issue, SERVIR-HKH has developed a climate resilient forest management system (CRFMS) to analyze how climate change can lead to forest degradation (Chap. 7). This information can be used for sustainable forest management by integrating it in the planning and management of forest ecosystems. Apart from analyzing the impact of climate change on the natural processes, CRFMS considers the various aspects of forest use and users.

One of the primary contributors to forest degradation in the region has been forest fires (Chap. 8). The fire-risk maps for Nepal developed by SERVIR-HKH has been useful for the development of a management plan based on the risk level of different districts. However, there are several limitations to the MODIS-based fire-monitoring mechanism due to its coarse resolution. Also, as in the case of Nepal, satellite-based observations do not really provide information on the damage caused by forest fires since most of these are understory fires. To capture and assess the fires and the damages they have caused, it is necessary to deploy a feedback system that collects information from the field. As for the larger $\mathrm{HKH}$ region, while the monitoring system has been useful in keeping an eye on fire incidence, a fire-risk forecasting system would be more effective in better planning of resources. In this regard, the capacity of the respective government agencies to operate the system is critical.

Our experiences show that partnerships and iterative engagements with user agencies from the very beginning are critical to ensuring the effective use of the monitoring system. In this context, the forest user groups should also give due weightage to the gender aspect in forest conservation. While the system was developed within the forest management context of Nepal, the efforts to outscale it in Myanmar have shown that the system can be useful across the region. However, this will require understanding different management contexts and identifying common issues through stakeholder consultations and participation. This will then pave for resilient forest management in the region and beyond.

\subsubsection{Water and Hydro-climatic Disasters}

Floods create havoc every year in the region during the monsoon season. There have been numerous efforts made by the national agencies and humanitarian organizations to improve upon the prior warning system for floods that can help save lives and 
properties. SERVIR-HKH has made efforts to enhance the flood early warning system in the region through its streamflow forecasting system (Chap. 9). The streamflow forecasting system has been highly effective in reducing the gap in flood forecasting in the region. For example, this regional system has provided discharge information for rivers without gauges which gave a better idea about transboundary flows in the case of Bangladesh. This system, based on a 15-day ECMWF (European Center for Medium-Range Weather Forecasts) projection, has produced very good results in capturing riverine flood. However, it has failed to capture events such as localized flash floods in the smaller catchments of the mid-hills. To address this issue, another forecast system, integrated with the High Impact Weather Assessment Tool (HIWAT) weather prediction model, has been developed, and this has shown the potential to capture such events (Chap. 12). For this purpose, systematic validation of the system with in situ data is crucial, but this has been a challenge since most of the small rivers do not have field stations. Another issue is access to such data which is limited due to the data policy and processing schedule of the respective agencies. Support from the Department of Hydrology and Meteorology (DHM) in Nepal and the Flood Forecasting and Warning Center (FFWC) in Bangladesh in the validation of the systems has been very useful. The experiences in the region show that coordination with the national agencies is crucial for the implementation of these systems; thus, it is important to build the confidence and capacity levels of the national agencies so that the system is used effectively.

Another information that is sought during flood events is on the extent of the flooded area; this information is required to support relief and response measures. The efforts of SERVIR-HKH in near-real-time mapping of inundation using SAR images (Chap. 10) have been effective in responding during flood events, especially in a context wherein cloud cover is a major limitation in the case of optical EO applications. These inundation maps are also highly accurate. Some of the challenges in terms of SAR have been about the availability of these images, the temporal frequency of data, and the time delay between the satellite acquisition of images and the availability of them for analysis. The SAR-based analysis also enables information on the water extent during a flood season; however, it cannot differentiate the floodwaters from the existing waterbodies and this hinders the mapping of the actual flood extent.

Another challenge is that while the system of streamflow prediction captures the rise and fall of water level within a stream, it is unable to provide any information on the damages to people and properties. Thus, the ultimate need is for a system that predicts flood extent and depth and also integrates population and socioeconomic data in it, thereby aiding in the assessment of damages and in the setting up of better response planning.

Under the thematic area of water and hydro-climatic disasters, another application undertaken by SERVIR-HKH has been the mapping of glaciers and glacial lakes of Afghanistan for the years 1990, 2000, 2010, and 2015 (Chap. 11). Being an arid and semiarid country, irrigation in Afghanistan is highly dependent on water from glacier melt. Thus, the assessment of glacier meltwater becomes important, also in terms of 
planning for hydropower. The impacts of climate change on the glacial environment have been one of the major concerns in the HKH region, especially Afghanistan. Thus, information on glacier area and volume, and changes over time are crucial for water resources and food security of Afghanistan. To address this gap SERVIR-HKH collaborated with the National Water Affairs Regulation Authority (NWARA) of Afghanistan to develop a glacier database for the country.

While the collaboration has led to the acquisition of glacier data for 1990-2015, at a five-year-intervals, the database needs to be duly updated and maintained with more recent data. More frequent annual data would also provide an option for analyzing glacier changes, along with other climatic variables. One of the technical challenges in the development of an updated glacier database is about identifying glaciers in areas with shadow, cloud, seasonal snow, or debris. This requires manual correction through visual interpretation, which is a labor-intensive process. One of the immediate applications of the time series glacial lake data is to identify the potentially dangerous glacier lakes in the country and to prioritize the riskiest glacial lakes depending on the physical and socioeconomic parameters for its continuous monitoring. A GLOF risk reduction strategy can also be established to reduce the risk and increase the resilience of the vulnerable communities. A policy to strengthen national and institutional capacities in order to implement GLOF-resilient development pathways will be very important for the country.

\subsubsection{Weather and Climate}

A reliable system to provide location-based forecast of extreme weather events like thunderstorm, hailstorm, and lightning has been needed in the region for a long time. The ensemble-based probabilistic forecast by HIWAT has been effective in filling some of the gaps in weather prediction in the HKH region (Chap. 12). The probability matched means from this ensemble forecast of precipitation also provides a more reliable prediction of high-intensity precipitation that might cause a flash flood in the mountainous river catchments. But while the forecast generated through HIWAT has shown a high degree of accuracy in predicting extreme weather, there is a lot of scope for improving the model and the configurations. As things stand, the data dissemination system now provides better access to forecast for meteorologists in the national agencies. But the current visualization system needs to be made easily understandable to the decision makers.

Currently, the system is implemented in NASA's SOCRATES, an HPC system set up for supporting the regional hubs. SERVIR-HKH's strategy for sustainability of this system has been to deploy it at the national agencies. However, the resources required for HPC is a significant bottleneck in this regard, even though the Bangladesh Meteorological Department (BMD) has made good progress in installing this system. Although SERVIR-HKH has organized several training events for the professionals from national agencies to use the system, more 
specialized training are required for the independent operation and maintenance of the system.

\subsection{Lessons from Crosscutting Areas}

SERVIR-HKH services are constantly guided by the intent to fill the major gaps and needs in the field of EO and geospatial applications in the region. The services have been developed to come up with the desired information products that can support decision-making at various levels. In this context, a number of crosscutting components play a crucial role in creating an enabling environment that can ensure the successful implementation of the services. These components are well identified in the service planning toolkit, and our experiences in these areas are presented below.

\subsubsection{Geoinformation Technology}

The approach of SERVIR-HKH toward selecting GIT solutions has been based on the institutional capacity of the partner agencies, and it has focused on the capacity enhancement of these agencies, thereby resulting in better adoption of the geoinformation system (Chap. 13). The glacier monitoring system in Afghanistan, streamflow forecasting in Bangladesh, and the National Land Cover Monitoring System in Nepal are some of the successful examples. SERVIR-HKH has also tried to improve the accessibility to these services by the rural communities who are constrained by poor internet connectivity and bandwidth limits. While the cloud-based processing system for a large volume of satellite data was a breakthrough in the development of land cover mapping and flood information services in the region, the existing capacity-in terms of IT infrastructure and technical skills - of the partner agencies in adopting these systems is still a major challenge. Besides, the rapid evolution of GIT and the need for maintenance and frequent updating of hardware and software have become critical constraints for the sustainability of these services.

\subsubsection{Capacity Building}

The capacity gap in the region was identified as one of the challenges for the successful implementation and use of geoinformation services. These challenges related to: selecting the right people from the partner organizations; keeping up to date with fast emerging and changing technologies; reducing the gender gap in participation; and ensuring the use of the learnings in actual operations. The 
multi-tier capacity development program of SERVIR-HKH, focusing on individuals and institutions, has produced very good results (Chap. 14). The combination of topic-based trainings in technologies and on-the-job trainings in system development and its use has been found to be useful in the successful co-development and deployment of the services in the partner agencies. Specialized trainings were conducted for the faculty of universities have been highly appreciated as they have also helped in developing a curriculum for geospatial science. Another highlight of the SERVIR-HKH training module to promote geospatial technologies has been its focus on women.

Our overall experience tells us that capacity building must be made a strong component of SERVIR-HKH service development and delivery. Our learning has also been that a more innovative approach needs to be adopted for reaching out to wider communities. The capacity building of the end user of the services is also vital to ensure the effective use of the systems. Besides, customized trainings and orientation programs for the higher management and policymakers are important for better adoption of these systems within the government agencies. In this regard, a number of events were also organized virtually during the COVID-19 phase, and this has given us new insights into online training approaches. While there were some limitations due to lack of face-to-face interactions, these virtual trainings successfully organised; also, they were cost-effective in terms of logistics and could accommodate a larger number of participants.

\subsubsection{Gender Inclusion}

Like other science and technology fields, there exists a considerable gender gap in the field of EO and GIT in the HKH region. There are far fewer women professionals in this field compared to men. There is a great need for improving women's access to geoinformation and their role in decision-making. For this, the services and products require customization for generating gender-inclusive information and building the capacity of women to use these products.

Gender inclusiveness is part of the SERVIR-HKH framework (Chap. 15). Our efforts to increase women's participation in capacity building through explicit women-focused events were successful in reaching out to more women. These events generated awareness as well as gave young women an opportunity to learn about the prospects of the GIT field. While some of the services could integrate gender and social issues, a lot more needs to be done in this area. A big issue in including more women professionals in the co-development process was that there is a lack of such professionals in the partner agencies. Targeted capacity building activities have to be carried out in this regard through universities and other relevant platforms. It is obvious that more gender-integrated studies as well as information products tailored for women and other marginalized groups would be helpful in extending the reach of these information services. 


\subsubsection{Communication}

Strategic communication (Chap. 16) is important for successful service design, delivery, and adoption. At the beginning of a service design process, communication is mainly internal and focused on ensuring that all the partners and stakeholders have the same knowledge and understanding of the products and services. At this stage, the communication activities aim to prepare materials on the product and services targeting the co-development partners to elaborate on the purpose and features of the services. At the later stages of the service delivery process, the communication activity focuses on the development of outreach materials targeting the end users. For effective communication, translation of some of the materials and interfaces in multiple languages are sometimes required. As communication is a continuous process, the materials need to be updated as per the progress of service development and delivery. While scientific articles or technical documentations are required for building the confidence of partners and the scientific community, descriptive materials are equally necessary for the wider users to build awareness about the services. The narration of success stories also helps to demonstrate the use and impact of the services. Besides developing communication materials, conducting regular communication campaigns is also crucial. These can be by way of technical and dissemination workshops, forums, newsletters, and social media campaigns. Constant engagement with media outlets is also an important facet of effective communication.

\subsubsection{User Engagement}

Systematic engagement with partners and stakeholders is an integral part of the service design and delivery process. The user engagement process within SERVIR-HKH has evolved over time and a dedicated user engagement mechanism was included in the service design and implementation from the beginning of the second phase (Chap. 17). The concept of co-design and co-development was adopted as a key strategy. Our experiences have been that the users are not homogeneous in terms of their situation, need, and capacity. For effective engagement, the collaboration strategy should be tailored to the situation of individual users. Continuous engagement with the key partners is also important and formal partnership agreements like MoU (Memorandum of Understanding) and LoI (Letter of Intent) are helpful in facilitating the process. Also, the involvement of various other stakeholders should not be overlooked. And it goes without saying that co-design and co-development are essential for the long-term sustenance of these services. 


\subsubsection{Monitoring and Evaluation}

Creating sustainable impacts in society through informed decision-making is the ultimate goal of SERVIR-HKH. The theory of change (ToC) approach adopted by SERVIR has been instrumental in aligning the service delivery process with outcomes and impacts on the ground (Chap. 18). The PIPA process has helped in building collective understanding between the SERVIR team and the partners for analyzing and developing the $\mathrm{ToC}$ for the services. This was important in terms of clarifying expectations and co-designing and implementing the services. This has also helped in the collective monitoring of progress and achievement. The PIPA process introduced at the service planning stage in the second phase has led to an improvement in aligning the service design and delivery components with user needs. However, PIPA, ToC, and MEL involve iterative processes and flexibility to adapt to the changing needs. For example, revisiting the $\mathrm{ToC}$ as per the project timeline helps partners to re-evaluate the products and services and thereby make the necessary adjustments. Moreover, defining clear indicators to track the changes in terms of outcomes and impacts, based on regular data collection and analysis, is important to ensure that the efforts are going in the right direction.

\subsection{Future Directions in EO Applications and Opportunities}

The opportunities for applications of EO in the HKH region are highly influenced by the global trends and the priorities set by the nations that are leading the space race. In addition to NASA as the leader in space exploration, the new strategy of the USA in deep space exploration has clearly identified the potential roles of the different government departments and the private sector (White House 2020). The achievements of SpaceX (www.spacex.com), a private company, in the development and reuse of orbital-class launch vehicles, thereby reducing substantial costs in the space industry, can be expected to bring a paradigm shift in the design and launching of EO satellites in the coming decades. Among the many planned missions of NASA, Landsat 9 is expected to provide continuity to the current monitoring applications, and NASA-ISRO Synthetic Aperture Radar (NI-SAR) will provide opportunities for many new applications on disasters, water resources, and vegetation monitoring (www.nasa.gov). Similarly, the Copernicus Space Component of the European Union has plans for expanding the Sentinel system to incorporate six high-priority missions: Anthropogenic $\mathrm{CO} 2$ Monitoring (CO2M); Land Surface Temperature Monitoring (LSTM); Polar Ice and Snow Topography Altimeter (CRISTAL): Copernicus Imaging Microwave Radiometer (CIMR); Copernicus Hyperspectral Imaging Mission for the Environment (CHIME); and the Radar Observing System for Europe in L-band (ROSE-L) (FutureEarth 2020). With the policy of Copernicus being to provide free access to Sentinel data, the EO 
community is highly optimistic about new and enhanced services in the areas of atmospheric, oceanic, cryospheric, and land global monitoring. There are also numerous space missions planned by Japan, Korea, China, India, and many other countries. Besides, the initiatives of the Committee on Earth Observation Satellites (CEOS) on international coordination of civil space-based EO programs in the development of compatible data products, formats, services, applications, and policies, and to optimize societal benefits, have been commendable in building collaborations and synergies. These efforts are also being supplemented by the Group on Earth Observation (GEO) with its many regional initiatives like AOGEO and thematic initiatives such as GeoGLOWS, GEOGLAM, GEOBON, and GEO Mountains, which are relevant to addressing the many concerns of the HKH region. The GEO has a dedicated initiative on EO for sustainable development (EO4SDG) to support the UN 2030 Agenda, in order to organize and help realize the potential of EO and enable societal benefits through the achievement of the SDGs (Anderson et al. 2017). In the context of all the HKH countries striving to achieve the SDGs, such initiatives provide frameworks and guidance on the applications of EO for various indicators.

Besides these trends in the field of EO, many disruptive technologies are progressing rapidly that will change the way we utilize EO data and information in the near future. To list a few of them, the Internet of things (IoT) will support billions of connected devices to sense the essential elements of our Earth environment and develop innovative paradigms for distributed computing. AI is another rapidly evolving technology that is increasingly being applied in the analysis of large volumes of EO data to extract meaningful information accurately and efficiently. The integrated use of AI, powerful cloud-based computing infrastructure, and new $5 \mathrm{G}$ connectivity with sensor networks in IoT is expected to bring in unprecedented opportunities at both local and global levels (Genderen et al. 2020).

In the context of the $\mathrm{HKH}$, we see many opportunities due to a huge gap in data, capacities, and services in EO and GIT, and also because of the increasing acceptance of these technologies by the national agencies as a means to improve the decision-making process. The signing of a declaration by the eight governments of the region supporting ICIMOD's HKH Call to Action during the "Hindu Kush Himalaya Ministerial Mountain Summit 2020" on 15 October 2020 shows a growing commitment to strengthening regional cooperation, promoting a united voice for the $\mathrm{HKH}$ at regional and global levels, and enhancing the uptake of scientific evidence for improving policies in the region on mountain environments and livelihoods (ICIMOD 2020a). Out of the six immediate actions identified by the call, Action 6 calls for promoting regional data and information sharing and science and knowledge cooperation in order to fill the data gaps and develop actionable knowledge that is mountain focused and HKH specific (ICIMOD 2020b). The efforts being made by SERVIR-HKH will directly contribute to addressing this call. SERVIR has also come up with its "SERVIR Strategic Plan 2020-2025" which states that one of its three strategic goals is to enhance its leadership power and influence globally. This reflects that the knowledge and experience generated by the SERVIR-HKH hub, in collaboration with its partners, have firmly established it as 
the regional center of excellence in the application of EO information. There is now growing confidence among the partners about SERVIR-HKH's applications and services. Indeed, the SERVIR network, with its many institutions worldwide and its regional hubs across the globe, provides a unique opportunity for cross-hub learning and working on innovative solutions using the current and future developments in EO and geospatial technologies.

\section{References}

Anderson K, Ryan B, Sonntag W, Kavvada A, Friedl L (2017) Earth observation in service of the 2030 agenda for sustainable development. Geo-spat Inf Sci. https://doi.org/10.1080/10095020. 2017.1333230

FutureEarth (2020) Plans for a new wave of European sentinel satellites. https://futureearth.org/ publications/explainers/plans-for-a-new-wave-of-european-sentinel-satellites/

Genderen Jv, Goodchild MF, Guo H, Yang C, Nativi S, Wang L, Wang C (2020) Digital earth challenges and future trends. In: Guo H, Goodchild MF, Annoni A (eds) Manual of digital earth. SpringerOpen, Berlin

ICIMOD (2020a) Hindu Kush Himalaya Ministerial Mountain Summit 2020 (https://www.icimod. org/hkhmms/)

ICIMOD (2020b) The HKH call to action to sustain mountain environments and improve livelihoods in the Hindu Kush Himalaya. ICIMOD, Kathmandu

White House (2020) A new era for deep space exploration and development, The white house national space council. https://www.whitehouse.gov/wp-content/uploads/2020/07/A-New-Erafor-Space-Exploration-and-Development-07-23-2020.pdf

Open Access This chapter is licensed under the terms of the Creative Commons Attribution 4.0 International License (http://creativecommons.org/licenses/by/4.0/), which permits use, sharing, adaptation, distribution, and reproduction in any medium or format, as long as you give appropriate credit to the original author(s) and the source, provide a link to the Creative Commons license, and indicate if changes were made.

The images or other third party material in this chapter are included in the chapter's Creative Commons license, unless indicated otherwise in a credit line to the material. If material is not included in the chapter's Creative Commons license and your intended use is not permitted by statutory regulation or exceeds the permitted use, you will need to obtain permission directly from the copyright holder.

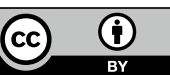

